05.1;06.5;10

\title{
Акустические колебания алюминиевых мембран при лазерном возбуждении по термоупругому механизму
}

\author{
(С) А.Л. Глазов, К.Л. Муратиков \\ Физико-технический институт им. А.Ф. Иофрфе РАН, Санкт-Петербург, Россия \\ E-mail: glazov.holo@mail.ioffe.ru
}

Поступило в Редакцию 14 февраля 2020 г.

В окончательной редакции 14 февраля 2020 г.

Принято к публикации 21 фревраля 2020 г.

\begin{abstract}
Предложена теоретическая модель формирования фотоакустического сигнала от металлических мембран лазерным излучением с учетом возбуждения в них дефектных состояний. Выполнено сравнение полученных теоретических результатов для алюминиевых мембран с толщиной $197 \mu \mathrm{m}$ в диапазоне частот от $20 \mathrm{~Hz}$ до $7 \mathrm{kHz}$ при их возбуждении лазерным излучением с длиной волны $660 \mathrm{~nm}$. Показано, что предложенная теоретическая модель обеспечивает хорошее совпадение экспериментальных и теоретических результатов для колебаний мембраны при чисто поверхностном характере поглощения лазерного излучения.
\end{abstract}

Ключевые слова: фотоакустика, мембраны, термоупругие колебания, дефекты, металлы.

DOI: 10.21883/PJTF.2020.10.49425.18247

Лазерные фотоакустические (ФА) исследования широко используются для изучения упругих, тепловых и оптических свойств разнообразных материалов. В настоящее время детально разработаны теоретические методы расчета процессов формирования ФА-сигналов в газовой среде, находящейся в контакте с исследуемым объектом, упругих колебаний и волн, возбуждаемых в твердотельных объектах и структурах. Полученные теоретические выводы неоднократно подтверждены результатами многочисленных экспериментов, выполненных на твердотельных объектах и жидкостях.

В последнее время большое внимание уделяется изучению особенностей проявления ФА-эффекта в различных мембранных и балочных структурах. Такие исследования выполнены для мембран и балок из полупроводниковых материалов [1,2], графена [3], металлов [4,5]. Для случая полупроводниковых материалов было показано, что учет особенностей процессов генерации и распространения носителей заряда лазерным излучением позволяет достаточно хорошо описать колебания таких мембран, возбуждаемых лазерным излучением по термоупругому механизму. Вместе с тем при объяснении экспериментальных данных, полученных для алюминиевых мембран в рамках традиционной термоупругой модели формирования акустических сигналов при лазерном возбуждении, имеются и определенные сложности.

В работе [5] были выполнены эксперименты на алюминиевых мембранах с толщинами от 112 до $280 \mu \mathrm{m}$. Акустические колебания мембран радиусом $4 \mathrm{~mm}$ возбуждались при равномерной засветке их поверхности излучением лазерного диода с длиной волны $660 \mathrm{~nm}$, модулированным во времени в диапазоне частот от $20 \mathrm{~Hz}$ до $7 \mathrm{kHz}$. Для уменьшения коэффициента отражения и увеличения эффективности возбуждения акустических колебаний облучаемая поверхность мембран покрывалась тонким слоем черной краски. Регистрация
ФА-сигнала осуществлялась методом открытой газомикрофонной ячейки. При этом считалось, что ФА-сигнал в ячейке возбуждается как за счет обычного теплового механизма, так и за счет изгиба мембраны. Для согласования экспериментальных и теоретических результатов в работе [5] потребовалось считать, что генерация тепловых волн в мембране носит объемный характер с эффективным коэффициентом поглощения лазерного излучения около $20 \mathrm{~mm}^{-1}$, который соответствует глубине проникновения излучения в алюминий около $50 \mu \mathrm{m}$. На наш взгляд, столь глубокое проникновение лазерного излучения в алюминиевую мембрану представляется маловероятным. В связи с этим целью настоящей работы является объяснение полученных в работе [5] результатов с учетом чисто поверхностного характера генерации тепловых волн на основе предложенной нами теоретической модели термоупругости металлов [6-8].

Основная особенность предлагаемой нами теоретической модели термоупругости металлов состоит в квазиравновесном возбуждении в нем дефектных состояний при лазерном воздействии с их последующей постепенной релаксацией в исходные состояния. Указанные переходы сопровождаются соответствующими изменениями свободной энергии металла [9] и объема его решетки. В соответствии с [9] присутствие в материале дефектов вызывает изменение его свободной энергии $F_{d}$ на величину

$$
F_{d}=K \Omega u_{l l} n
$$

где $K$ - модуль всестороннего сжатия, $\Omega$ - дилатационный параметр, характеризующий изменение объема кристалла при образовании в нем дефекта, $u_{i k}$ - тензор деформации решетки, $n$ - концентрация дефектов.

При анализе экспериментальных результатов работы [5] следует учесть, что они были выполнены при низких частотах. Поэтому для расчета колебаний мембран 


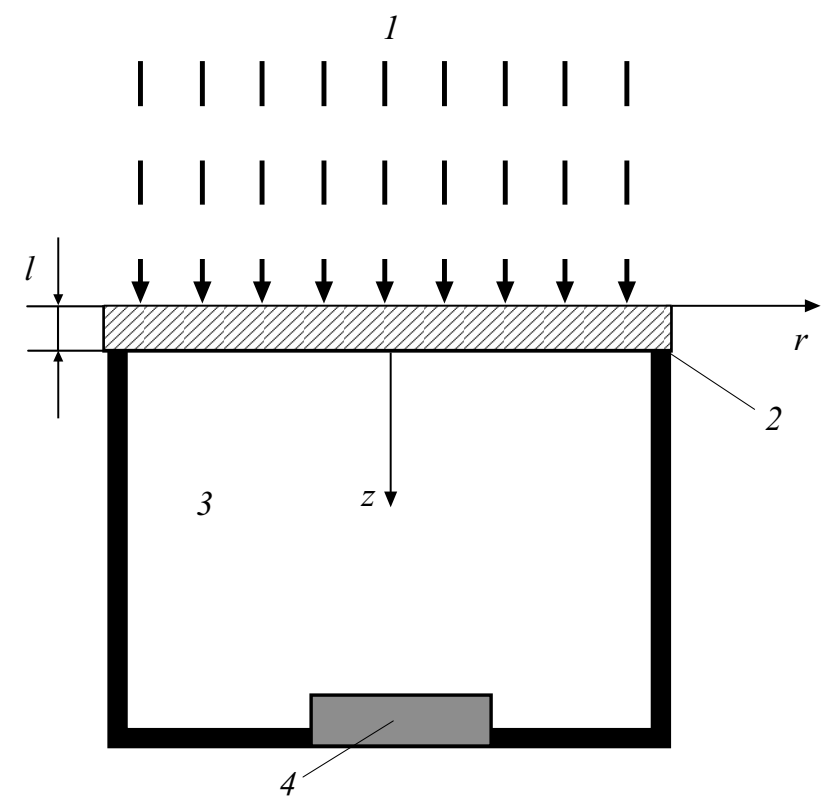

Рис. 1. Схема эксперимента. 1 - греющее лазерное излучение, модулированное по интенсивности с переменной частотой $f$; 2 - алюминиевая мембрана; 3 - фотоакустическая камера; 4 - микрофон.

можно воспользоваться квазистатическим приближением. Тогда с учетом соотношения (1) уравнения движения из работ $[4,5]$ для расчета компонент вектора смещения мембраны в цилиндрических координатах можно записать в виде (ось $z$ направлена перпендикулярно поверхности мембраны)

$\Delta u_{r}-\frac{u_{r}}{r^{2}}+\frac{1}{1-2 v} \frac{\partial e}{\partial r}+\frac{2(1+v)}{1-2 v}\left(\Omega \frac{\partial n}{\partial r}-\alpha_{T} \frac{\partial T}{\partial r}\right)=0$,

$$
\Delta u_{z}+\frac{1}{1-2 v} \frac{\partial e}{\partial z}+\frac{2(1+v)}{1-2 v}\left(\Omega \frac{\partial n}{\partial z}-\alpha_{T} \frac{\partial T}{\partial z}\right)=0,
$$

где $\Delta-$ оператор Лапласа, $e=u_{r r}+u_{\theta \theta}+u_{z z}$, $u_{\theta \theta}=u_{r} / r, \alpha_{T}$ - коэффициент теплового расширения материала.

Система уравнений (2) аналогично работам [4,5] решалась при граничных условиях, соответствующих закрепленному краю мембраны и свободным боковым поверхностям (рис. 1). Кроме того, поскольку в экспериментах [5] осуществлялась равномерная засветка мембраны, распределение температуры в ней считалось не зависящим от координаты $r$.

Уравнения (2) отличаются от уравнений работ $[4,5]$ наличием слагаемых с градиентом концентрации дефектов. Динамика концентрации дефектов в металле может быть описана с помощью уравнения баланса частиц с функцией источника в виде закона Аррениуса [6-8]:

$$
\frac{\partial n}{\partial t}+\frac{n}{\tau}=J
$$

где $\tau$ - время релаксации дефектов, $J$ - объемный источник дефектов, возбуждаемых лазерным излучением, определяемый законом Аррениуса.
В рамках настоящей работы предполагается, что генерация дефектов в материале происходит за счет модуляции температуры при поглощении лазерного излучения. Расчет их концентрации для этого случая приведен в работах $[7,8]$. При поверхностном характере поглощения мембраной лазерного излучения, модулированного во времени с круговой частотой $\omega$, и при пренебрежении теплоотводом от нее в окружающую воздушную среду ее температура определяется выражением

$$
T(z, \omega)=\frac{I_{0}}{k} \frac{\operatorname{ch}\left[\sigma\left(\frac{l}{2}-z\right)\right]}{\sigma \operatorname{sh}(\sigma l)},
$$

где $I_{0}-$ интенсивность излучения лазера, поглощенного мембраной; $k$ - теплопроводность материала мембраны, $\sigma=\sqrt{i \omega / \kappa}, \kappa-$ температуропроводность мембраны.

Интерпретацию экспериментальных результатов работы [5] необходимо проводить с учетом двух меха-
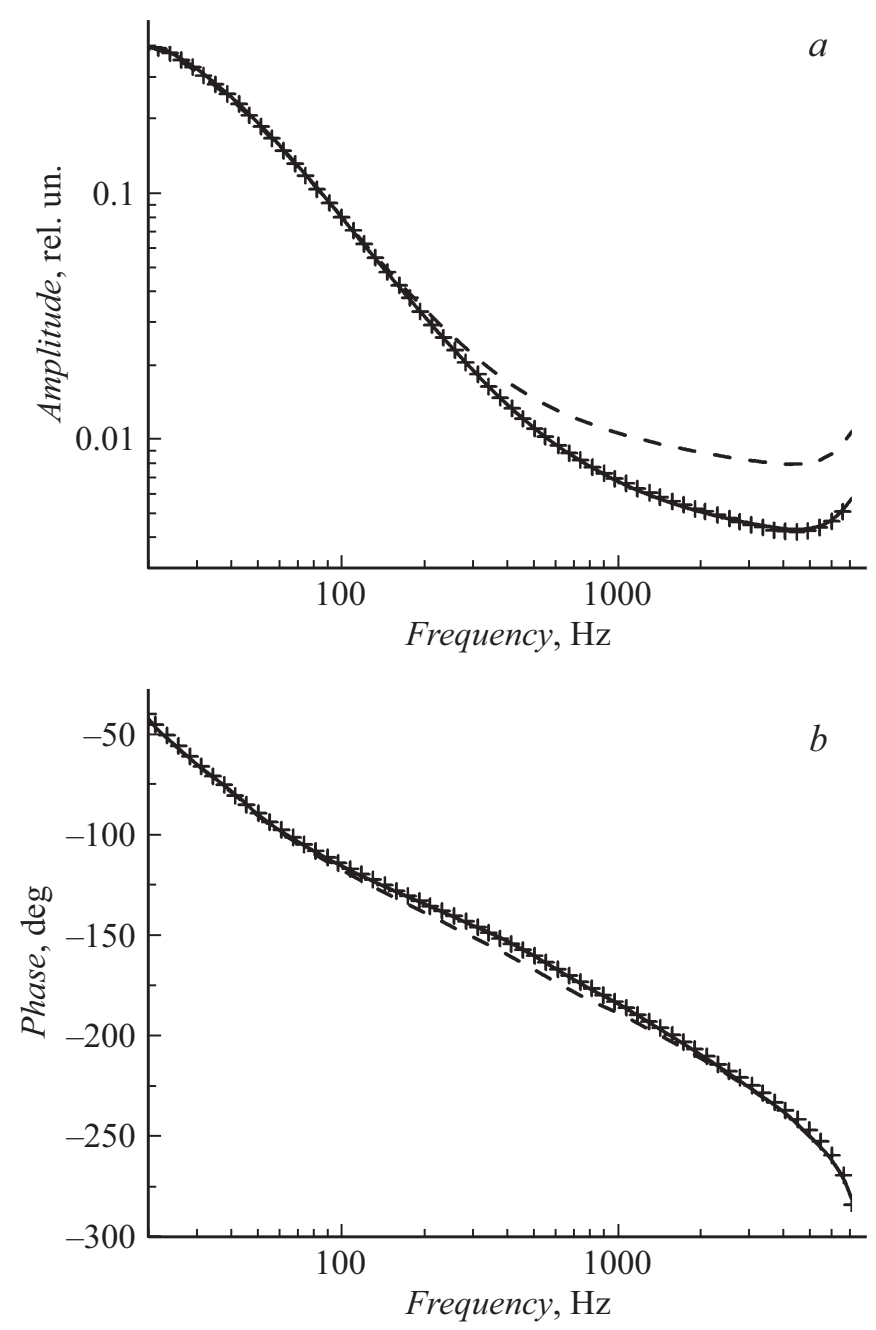

Рис. 2. Амплитуда $(a)$ и фаза $(b)$ фотоакустического сигнала от непрозрачной мембраны толщиной $197 \mu \mathrm{m}$. Крестики - экспериментальные данные из работы [5], сплошная кривая - расчет при $\alpha_{T \text { eff }}=11.8 \cdot 10^{-6} \mathrm{~K}^{-1}$, штриховая кривая - расчет при справочном значении $\alpha_{T}=23 \cdot 10^{-6} \mathrm{~K}^{-1}$. 
низмов генерации ФА-сигнала. Первый из них является чисто тепловым и определяется нагревом воздуха вблизи мембраны, а второй - чисто акустическим, обусловленным ее изгибом при термоупругих деформациях. В работе [7] было показано, что в линейном по температуре приближении влияние генерации дефектов на термоупругие деформации в металле при лазерном облучении может быть учтено введением некоторого эффективного коэффициента теплового расширения. С учетом указанных обстоятельств аппроксимация экспериментальных данных, взятых из работы [5], проводилась при двух варьируемых параметрах: общем множителе и коэффициенте теплового расширения. При этом минимум суммы квадратов отклонения $s$ теоретических результатов от экспериментальных данных искался одновременно для логарифмов синфазной и квадратурной составляющих сигнала. Для непрозрачного образца (коэффициент поглощения света $\beta$ больше $1000 \mathrm{~mm}^{-1}$ ) минимум составлял $s=0.15$ при эффективном значении коэффициента теплового расширения $\alpha_{\text {eff }}=11.8 \cdot 10^{-6} \mathrm{~K}^{-1}$. Экспериментальные зависимости и аппроксимирующие их теоретические кривые для амплитуды и фазы ФА-сигнала приведены на рис. 2. Там же штриховой линией показана зависимость сигнала для алюминия со стандартными характеристиками. Для сравнения, используя подход, предложенный в [5], т. е., варьируя коэффициент поглощения света образцом при постоянном табличном коэффициенте теплового расширения для алюминия $\alpha_{T}=23 \cdot 10^{-6} \mathrm{~K}^{-1}$, получаем минимум суммы квадратов отклонения $s=0.80$ при $\beta=20.8 \mathrm{~mm}^{-1}$. При аппроксимации только амплитуды сигнала находим соответственно для двух подходов $\quad \alpha_{\text {e eff }}=11.8 \cdot 10^{-6} \mathrm{~K}^{-1} \quad(s=0.0095)$ или $\beta=15.6 \mathrm{~mm}^{-1}(s=0.019)$ при $\alpha_{T}=23 \cdot 10^{-6} \mathrm{~K}^{-1}$, т.е. при варьировании коэффициента теплового расширения получается одинаковая величина для аппроксимации как полного набора данных, так и отдельно амплитуды сигнала с меньшей суммой квадратов отклонений, чем при варьировании коэффициента поглощения света.

Таким образом, представленные теоретические результаты показывают, что учет формирования динамических дефектных состояний в металле при лазерном облучении позволяет достаточно хорошо описать экспериментальные результаты по возбуждению акустических колебаний в алюминиевых мембранах при чисто поверхностном характере поглощения излучения.

\section{Конфликт интересов}

Авторы заявляют, что у них нет конфликта интересов.

\section{Список литературы}

[1] Sievila P., Chekurov N., Raittila J., Tittonen I. // Sensors Actuators A. 2013. V. 190. P. 90-95. http://doi.org/10.1016/j.sna.2012.11.020

[2] Coutu R.A., Jr., Medvedev I.R., Petkie D.T. // Sensors. 2016. V. 16. N 2. P. 251 (1-11). https:// doi:10.3390/s16020251
[3] Zelinger Z., Jandai P., Suchanek J., Dostal M., Kubat P., Nevrly V., Bitala P., Civis S. // J. Sensors Sensor Syst. 2015. V. 4. N 1. P. 103-109. https:// doi:10.5194/jsss-4-103-2015

[4] Rousset G., Lepoutre F., Bertrand J. // J. Appl. Phys. 1983. V. 54. N 5. P. 2383-2391. https://doi.org/10.1063/1.332352

[5] Markushev D.D., Ordonez-Miranda J., Rabasovic M.D., Galovich S., Todorovich D.M., Bialkovski S.E. // J. Appl. Phys. 2015. V. 117. N 24. P. 245309 (1-8). DOI: 10.1063/1.4922718

[6] Морозов Н.Ф., Муратиков К.Л., Семенов Б.Н., Индейцев Д.А., Вавилов Д.С. // ДАН. 2019. Т. 485. № 4. С. 438-441.

[7] Глазов А.Л., Муратиков К.Л. // Письма в ЖТФ. 2019. Т. 45. B. 17. C. $51-54$.

[8] Глазов А.Л., Морозов Н.Ф., Муратиков К.Л. // Письма в ЖТФ. 2020. Т. 46. В. 4. С. 22-25.

[9] Косевич А.М. Физическая механика реальных кристаллов. Киев: Наук. думка, 1981. 328 с. 